\title{
Application of Radon Transforms and Time-Frequency Representations to ISAR Imagery
}

\author{
Philippe Steeghs and Sjoerd J. Gelsema \\ TNO Physics and Electronics Laboratory \\ Oude Waalsdorperweg 63, 2509 JG, The Hague, The Netherlands
}

\begin{abstract}
The use of ISAR imagery for Automatic Target Recognition is seriously hampered by the difficulty of target motion compensation. Phase perturbations that result from target maneuvers during the processing interval need to be corrected for. In a previous paper, we demonstrated the use of the local Radon transform for estimating the radial velocity of a target. This estimate can then be used to align a sequence of range profiles prior to cross-range compression. In this paper, we make a quantitative comparison of the results that are obtained using different types of local Radon transformations. In the second part of this paper we outline an algorithm for compensation of phase perturbation that are caused by non-uniform target rotation. The algorithm has been tested on simulated data.
\end{abstract}

Keywords: ISAR, motion compensation, time-frequency analysis, Radon transformation

\section{INTRODUCTION}

Traditionally, ISAR images are formed by evaluating the phase history of a sequence of range profiles during a certain coherent processing interval, using the Fourier transformation. However, the use of this method for radar target recognition is seriously hampered by the difficulty of target motion compensation. Phase perturbations imposed by target maneuvers during the processing interval need to be corrected for.

In a previous paper, we described the use of the the Radon transform of the time-frequency representation for the correction of phase perturbations during the coherent processing interval. ${ }^{1}$ In addition, we demonstrated the application of the local Radon transform to the estimation of the radial velocity of the target. The estimated radial velocity was then used for alignment of the range profiles prior to cross-range compression.

In this paper we further explore these two issues. In the first part of the paper we discuss range alignment using the - Cohen's class - quadratic local Radon transform for radial velocity estimation. In the second part an algorithm for compensating for non-uniform target rotation is demonstrated. The algorithm is based on the detection of chirp signal components in the Radon transform of the time-frequency representation. In an iterative procedure we then determine the location of scatterers that are associated with the chirp components in the signal. The algorithm can be considered a modification of the CLEAN algorithm for target feature extraction. $^{2}$ With the CLEAN algorithm sinusoids are extracted, whereas our algorithm extracts chirps.

\section{TARGET TRANSLATIONAL MOTION}

In ISAR range-Doppler imaging with ground-based radar systems the radar location is fixed and the synthetic aperture is created by the motion of the target. Figure la shows the geometry of ground-to-air radar ISAR imaging. The (simulated) data that is used throughout this paper has been generated using the method of Chen and Miceli. ${ }^{3}$ In order to obtain simulated data from a maneuvering target a sequence of range profiles is computed. The individual range profiles are obtained by summation of a series of time-delayed point scatterer responses. The motion of the target is introduced into the model by changing the positions of the scatterers in subsequent range profiles.

This research was funded in part by Office of Naval Research NICOP grant N000140110345. Further author information: Address: TNO Physics and Electronics Laboratory, Radar Systems and Information Extraction, Oude Waalsdorperweg 63, 2509 JG, The Hague, The Netherlands, E-mail: \{steeghs,gelsema\}@fel.tno.nl, Telephone: +31703740000 
(a)

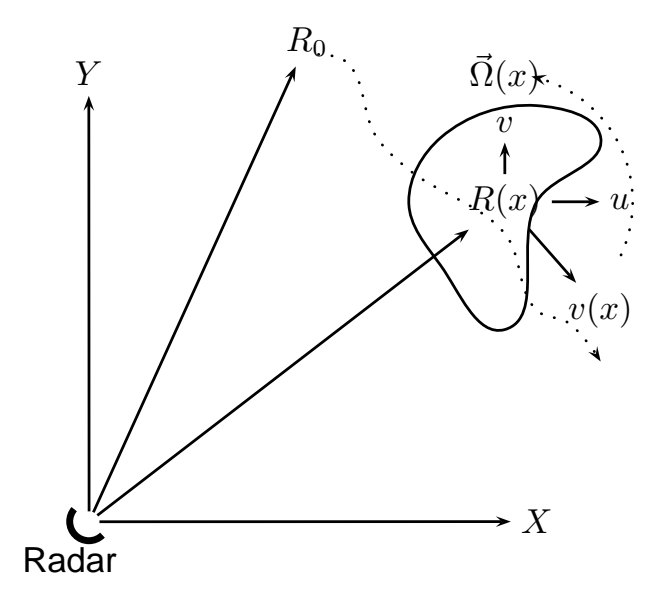

(b)

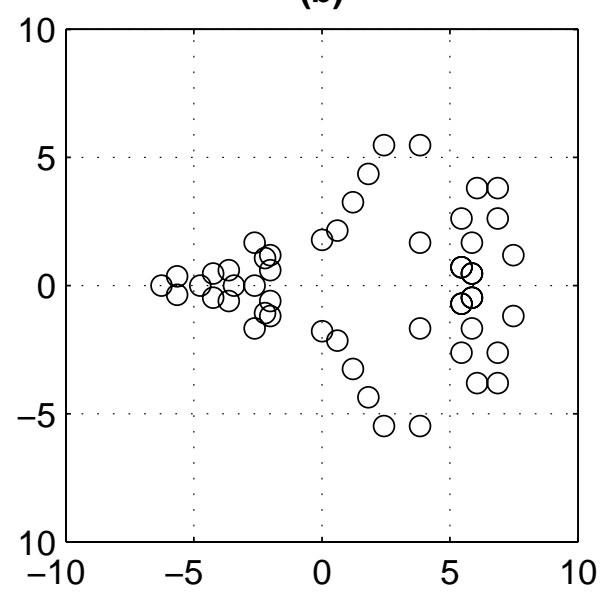

Figure 1: (a) Radar and target geometry, and (b) target model.

The radar is located at the origin of the radar coordinate system, with axes $X$ and $Y$. At $X=0$ the target is located at range $R_{0}$. Due to translation of the target with time-varying target velocity $v(x)$, the target location shifts to $R(x)$ (we denote the cross-range time coordinate by $x$ ). The target coordinate system, with coordinates $(u, v, w)$, has its origin at the center of rotation of the target.

The phase of a radar signal that has been reflected by a point scatterer at the center of rotation of a moving target at range $R(x)$ is given by $^{4}$

$$
\phi(x)=2 \pi \frac{2 f}{c}\left(R_{0}-\int_{x=0}^{x} v_{R}(x) d x\right)
$$

where $c$ is the speed of wave propagation, $f$ is the frequency of the transmitted radar signal, $R_{0}$ is the range of the target rotation center at $t=0$, and $v_{R}(x)$ is the target velocity with respect to the Line-Of-Sight (LOS) of the radar. The LOS velocity $v_{R}(x)$ determines the Doppler frequency shift of the the rotation center of the target and is defined as

$$
v_{R}(x)=v(x) \cdot \vec{l}
$$

where $v(t)$ is the velocity of the target and $\vec{l}$ is the LOS unit vector. ${ }^{5}$ The purpose of ISAR motion compensation is to remove the effect of the target radial velocity on the Doppler frequency, such that only the Doppler shift that results from target rotation remains.

In our simulations we have modeled the target modeled as a collection of $n$ point scatterers, with coordinates $\left(u_{i}, v_{i}, w_{i}\right), i=1, \ldots n$, in the 3 -D target coordinate system. Figure $1 \mathrm{~b}$ shows the geometry of a target projected on the $(u, v)$-plane. The output of the simulation is a sequence of range profiles $u(x, r)$, where the variable $r$ is used to denote the range time coordinate and $x$ to denote the pulse (slow) time.

\section{THE LOCAL RADON TRANSFORMATION}

The linear Radon transformation of a 2-D signal $u(x, r)$ is defined as

$$
\breve{u}(p, \tau)=\int_{x=-\infty}^{+\infty} u(x, r=\tau+p x) d x .
$$

In this parameterization the Radon transform performs the integration of the 2-D signal along slanted lines with intercept $\tau$ and direction $p$. If the a target moves with a constant radial target velocity $v_{R}(x)=v_{0}$ the peak 
(a)

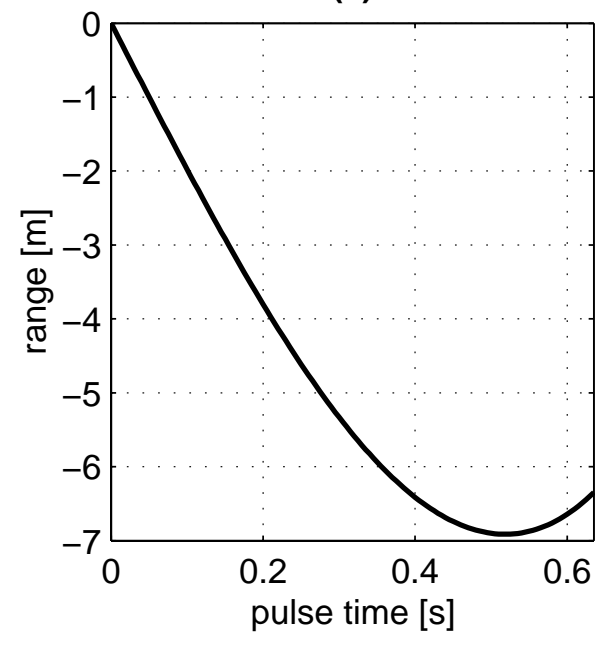

(b)

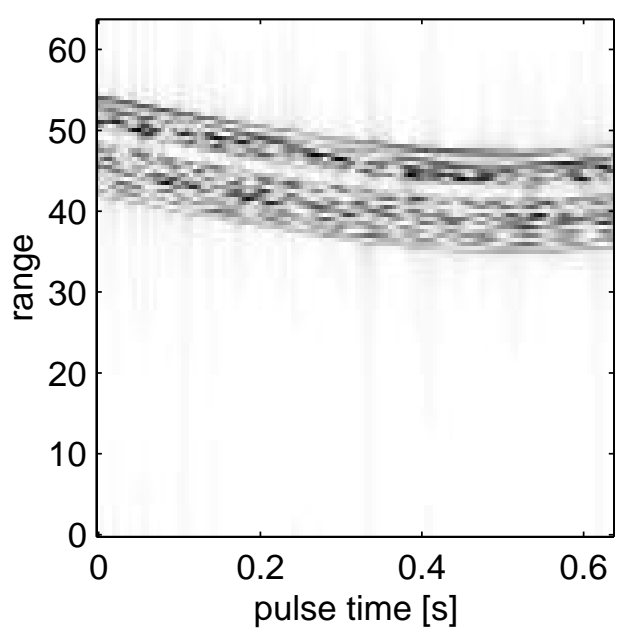

Figure 2: (a) Flight path, (b) range profiles.

of the Radon transform of a set of range profiles envelopes can be used to estimate $v_{0}$. Subsequently the range profiles can be aligned using the radial velocity estimate. ${ }^{6}$ Figure $2 \mathrm{~b}$ shows a set of simulated range profiles that is obtained with a time-varying velocity $v_{R}(x)=v_{0}+\alpha_{v} x^{2}$. The radial flight path $R(x)$ of the target is shown in Fig. 2a. The global Radon transform is not suited for the estimation of a time-varying radial target velocity $v_{R}(x)$. A local measurement of the slope of the range profile envelopes can be obtained using a local Radon transformation. ${ }^{1}$ Using the relation between the Fourier transformation and the Radon transformation, we can define a class of local Radon transformations that is an extension of the Cohen's class of quadratic time-frequency representations for time-varying spectral analysis ${ }^{7-9}$.

The straightforward approach to devise a local Radon transformation is to divide the data into overlapping segments and perform the Radon transformation on each of the segments. Let the signal $u_{w}(x, r)$ be a portion of the data weighted by the sliding window function $w(x)$ centered at cross-range time $x$, i.e.

$$
u_{x}\left(x^{\prime}, r\right)=u\left(x^{\prime}, r\right) w\left(x^{\prime}-x, r\right) .
$$

The sliding-window Radon transform is then given by

$$
\breve{u}_{x}(p, \tau)=\int_{x^{\prime}=-\infty}^{+\infty} u_{x}\left(x^{\prime}, r=\tau+p x^{\prime}\right) d x^{\prime} .
$$

The sliding-window Radon transform and the sliding-window Fourier transform are closely related. Taking the Fourier transformation of Eq. (5) with respect to the range $r$, we find

$$
\hat{u}_{x}(p, f)=\int_{x^{\prime}=\infty}^{+\infty} e^{j 2 \pi f p x^{\prime}} \hat{u}_{x}\left(x^{\prime}, f\right) d x^{\prime},
$$

which is a sliding-window Fourier transformation with respect to the $x$-coordinate. This relation can now be used to devise a class of local Radon transformations analoguous to the Cohen's class of time-frequency representations.

A fundamental time-frequency representation in Cohen's class is the Wigner distribution, defined as ${ }^{7}$

$$
W(t ; f)=\int_{\tau=-\infty}^{+\infty} e^{-j 2 \pi f \tau} u(t+\tau / 2) u^{*}(t-\tau / 2) d \tau,
$$


which is the Fourier transformation with respect to the shift variable $\tau$ of the instantaneous auto-correlation function $R(t ; \tau)$, given by

$$
R(t ; \tau)=u(t+\tau / 2) u^{*}(t-\tau / 2)
$$

where the asterisk denotes complex conjugation. The Wigner distribution provides a sharper time-frequency localization than the sliding-window Fourier power spectrum. However, the quadratic structure of the Wigner distribution gives rise to cross terms, which may seriously complicate the interpretation of the Wigner distribution. The cross terms can be suppressed by smoothing the Wigner distribution over time and frequency to obtain a new representation

$$
P(t ; f)=\int_{t^{\prime}=-\infty}^{+\infty} \int_{f^{\prime}=-\infty}^{+\infty} \Psi\left(t^{\prime} ; f^{\prime}\right) W\left(t-t^{\prime} ; f-f^{\prime}\right) d t^{\prime} d f^{\prime}
$$

The smoothing function $\Psi(t ; f)$ is called the kernel of the time-frequency representation $P(t ; f)$. The class of the time-frequency representations that can be obtained by smoothing the Wigner distribution in time and frequency is called Cohen's class. The sliding-window power spectrum or spectrogram is a member of Cohen's class. The kernel of the spectrogram is given by

$$
\Psi_{\operatorname{SPEC}}(t ; f)=W_{w}(-t ;-f),
$$

where $W_{w}(t, f)$ is the Wigner distribution of the sliding window function $w(t)$.

The properties of a certain Cohen's class quadratic time-frequency representation are determined by the corresponding kernel function. In general, the kernels are most easily designed in the 2-D Fourier transformed domain. The 2-D Fourier transform of the 2-D convolution Eq.(9) is given by

$$
M(\nu ; \tau)=\Psi(\nu ; \tau) A(\nu ; \tau)
$$

where $M(\nu ; \tau)$ is the characteristic function of the time-frequency representation and $A(\nu, \tau)$ is the ambiguity function of the signal $u(t)$.

Using the relation between local Radon transformation and the sliding-window Fourier transformation Eq.(6) we now propose a class of quadratic local Radon representations. The relation of the quadratic local Radon representation and sliding-window Radon power spectrum Radon transform analogue of the relation between Wigner distribution and spectrogram.

The local Wigner-Radon representation of the 2-D signal $u(x, r)$ is defined as

$$
S(x, r ; p, \tau)=\int_{\xi=-\infty}^{+\infty} R(x, r ; \xi, \rho=\tau+p \xi) d \xi .
$$

This local Radon representation is obtained by integration along slanted lines of the 2-D instantaneous autocorrelation function $R(x, r ; \xi, \rho)$, defined as

$$
R(x, r ; \xi, \rho)=u(x+\xi / 2, r+\rho / 2) u^{*}(x-\xi / 2, r-\rho / 2) .
$$

As a result, we can apply the tools and methods of quadratic time-frequency analysis to local Radon power spectrum analysis. For instance, cross term suppression can be achieved by smoothing the Wigner-Radon representation. A similar approach is taken as in standard quadratic time-frequency analysis. Kernels that have desirable properties, such as optimal cross term suppression for a broad class of signals, can be also be employed for local Radon transform analysis. The corresponding local Radon power spectra have similar properties as their time-frequency counterpart. 


\section{RANGE ALIGNMENT USING THE QUADRATIC LOCAL RADON TRANSFORMATION}

In Fig. 3 we compare the results of Quadratic Local Radon Transform (QLRT) analysis of the range profiles of Fig. 2 with four different kernels. There is only one target present in the range profile sequence. As a result, the range shift due to the translational motion is constant in range. Therefore, a local analysis in the $\mathrm{x}$-coordinate only is sufficient in the single target case. The images in Fig. 3 represent the local directional decomposition $S(x ; p, \tau=0)$ of the range profile envelopes $|u(x, r)|$. Figure $4 \mathrm{~b}$ shows the estimate of the local direction of the range profile envelopes $p_{m}(x) \approx \mathrm{d} R(x) / \mathrm{d} x$. The local direction is directly proportional to the radial target velocity $v_{R}(x)$ and can be estimated as the location of the peak of $S(x ; p)$. Figure 4 a shows the estimated radial flight path of the target $R(x)$.

The spectrogram kernel LRT (cf. Eq.(10)) suffers from similar drawbacks as the spectrogram. The extracted maximum deviates strongly from the true direction where sudden changes in the signal occur. The results of the Choi-Williams kernel LRT with kernel

$$
\Psi_{\mathrm{CW}}(\kappa ; \xi)=e^{-\frac{(2 \pi \kappa \xi)^{2}}{\sigma}}
$$

where $\kappa$ and $\xi$ are the Doppler frequency and pulse time correlation variables, and Smoothed Pseudo Wigner LRT with kernel

$$
\Psi_{\mathrm{SPW}}(\kappa ; \xi)=h(\kappa) w(\xi / 2) w^{*}(-\xi / 2),
$$

are closest to the true direction of the target (Figs.3b and 3c). The result of the Cone-kernel LRT, with

$$
\Psi_{\mathrm{CK}}(\kappa ; \xi)=w(\xi)|\xi| \frac{\sin (\pi \kappa \xi)}{\pi \kappa \xi},
$$

is close to that of the spectrogram kernel LRT.

Figure 5 shows the ISAR images that are obtained when using the different QLRT kernels for range alignment. The Doppler-range ISAR image $u\left(f_{D}, r\right)$ is obtained by taking the DFT with respect to the pulse time $(x)$ of the aligned range profiles. A measure of the quality of the range alignment is given by the peakedness of the images (after scaling to their peak amplitude), defined as

$$
k=\sum\left|u\left(f_{D}, r\right)\right|^{4} .
$$

The images show that best focussing is achieved with the Choi-Williams kernel LRT and Pseudo Smoothed Wigner kernel LRT. When using the true radial flight path for range alignment we have $k=13.2$. We have tested our range alignment procedure on measured data, with similar results and relative performance of the different kernels.

\section{DOPPLER FREQUENCY ALIGNMENT}

After compensation for linear motion of the target, the remaining phase variations in a range cell during the CPI are due to the rotational motion of the target. In traditional range-Doppler ISAR imaging uniform target rotation is assumed, since it will result in the localization of point scatterers in the target at a single Doppler frequency. The Doppler frequency produced by a scatterer in the target can be expressed as ${ }^{4}$

$$
f_{D}(x)=\frac{2 f}{c}|\vec{v}(x) \cdot \vec{l}(x)|
$$

where $\vec{v}(t)$ is the scatterer's velocity vector. Let $\vec{r}=(u, v, w)$ be the position vector of a scatterer measured from the center of rotation, the Doppler frequency shift of the scatterer is then given by ${ }^{4,5}$

$$
f_{D}(x)=\frac{2 f}{c}(\vec{\Omega}(x) \times \vec{r}) \cdot \vec{l}(x) .
$$


(a)

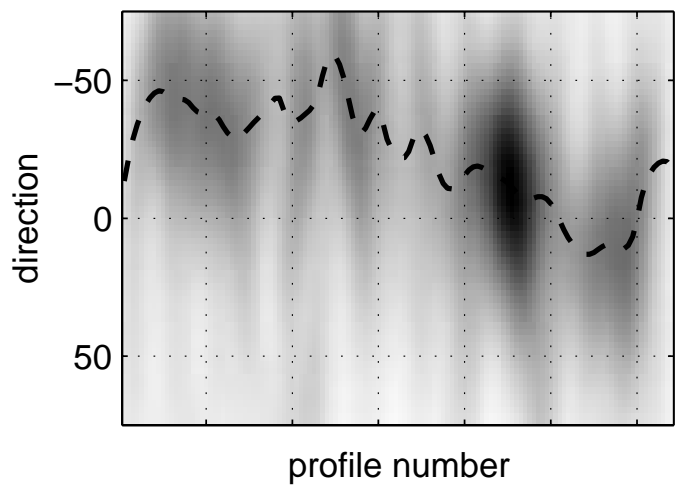

(c)

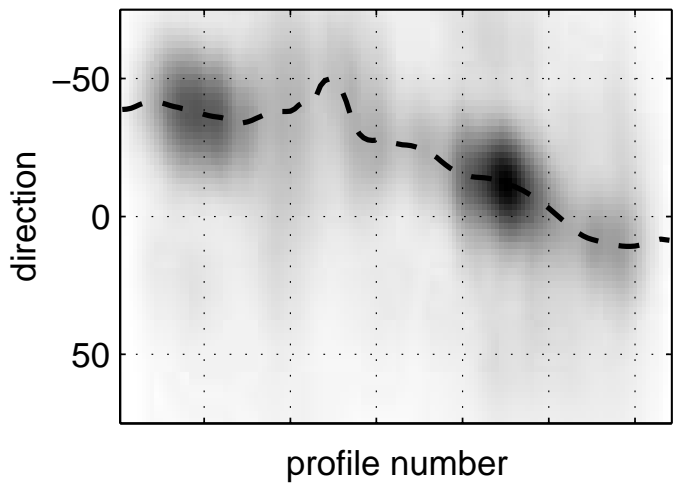

(b)

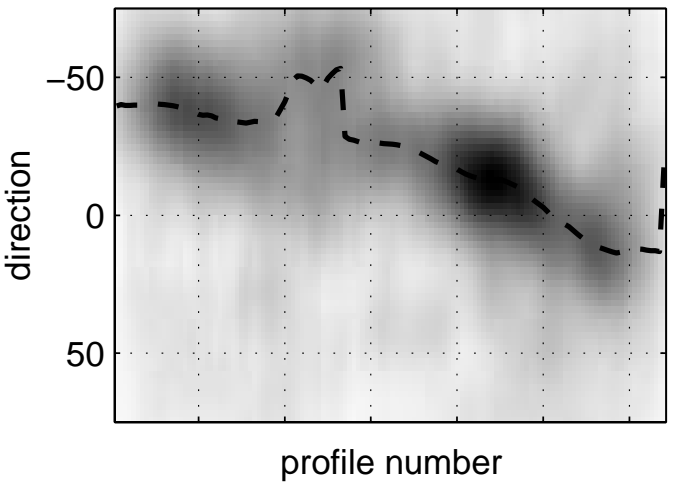

(d)

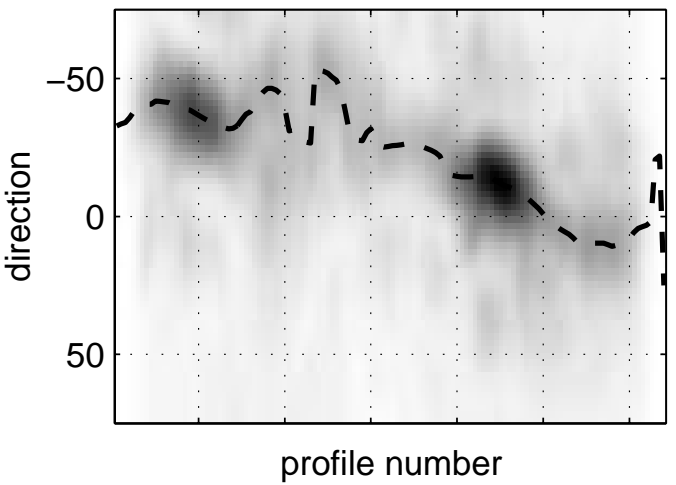

Figure 3. Local directional spectra, (a) Spectrogram kernel, (b) pseudo smoothed Wigner distribution kernel, (c) Choi-Williams kernel, (d) cone kernel. The dashed line is the location of the maximum.

For a smoothly moving target, the angle $\eta$ between the rotation vector $\vec{\Omega}$ and the LOS unit vector remains approximately constant over the CPI. The Doppler frequency shift that is induced by the target rotation is constant as well, and is given by ${ }^{4}$

$$
f_{D}=\frac{2 f}{c} \Omega \sin (\eta) r_{c}=\frac{2 f_{c}}{c} \Omega_{e} r_{c}
$$

with $\Omega_{e}$ the magnitude of the effective rotation vector and $r_{c}$ the cross range displacement of the scatterer. The rotation vector is a combination of the roll, pitch, and yaw rotations of the target. The combined actual rotation vector determines the Doppler frequency-shift of the scatterers. ${ }^{4,5}$ The effective rotation vector $\vec{\Omega}_{e}$ is normal to the LOS unit vector and normal to the image projection plane. ${ }^{5}$

For non-uniform target rotation the Doppler frequency will not remain constant during the CPI and as a result the ISAR image will be blurred. Time-frequency analysis has been proposed to capture the variations in Doppler frequency as a function of time. ${ }^{10,11}$ Although time-frequency analysis is has proven itself as an excellent tool for analyzing time-varying Doppler frequencies, there are some drawbacks that decrease its usefulness for ISAR target recognition. For instance, the improved resolution of time-frequency representations of Cohen's class seldomly completely outweighs the problem of cross term interference in the time-frequency images. Moreover, time-frequency analysis expands a 2-D sequence of range profiles into a 3-D sequence of ISAR images, and the amount of data may quickly become difficult to handle for a target recognition algorithm.

However, for certain types of Doppler frequency variation the time-frequency representation can be used as an intermediate step for coherent processing of non-stationary signals. In the following we will demonstrate this approach for a target with accelerating rotation. We have developed an algorithm to correct for this type of 
(a)

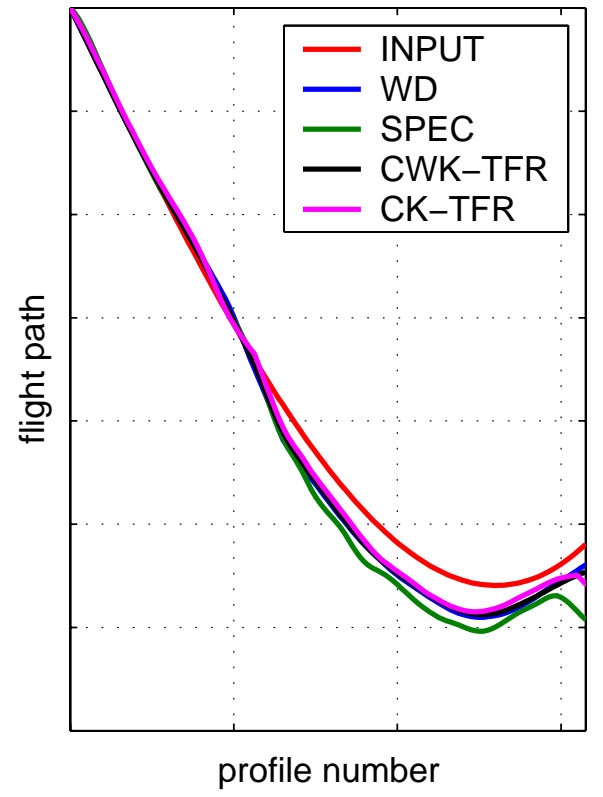

(b)

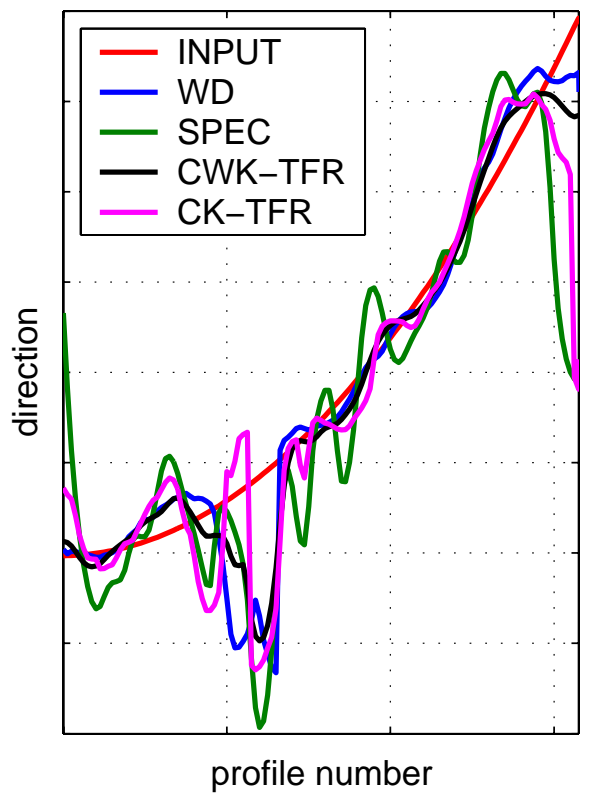

Figure 4: (a) Flight path, (b) local direction.

non-uniform rotation using the linear Radon transformation of the time-frequency representation of a sequence of range profiles.

\section{RADON-WIGNER CHIRP CLEAN ALGORITHM}

In Figs. 6a and 6b we show a two point scatterer target model and the simulated radar return signal from this target, where we have increased the (yaw) rotation rate linearly during the CPI. The angle $\eta=\pi / 2$ between the rotation vector $\vec{\Omega}$ and the LOS unit is assumed to remain constant over the CPI. The Doppler frequency shift for a single point scatterer that is induced by accelerating rotation can now be approximated by

$$
f_{D}(x)=\frac{2 f}{c}\left(\Omega_{0}+\alpha_{\Omega} x\right) r_{c}=f_{D}(x=0)+\alpha_{f_{D}} x,
$$

where $\alpha_{\Omega}$ is the acceleration rate of the rotation. The time-frequency representation $P\left(x, f_{D}\right)$ of the radar return signal is shown in Fig. 6c. The two point scatterers are expressed as two ridges with in time-frequency representation with intercept $\nu_{D}=f_{D}(x=0)$ and (constant) direction $\alpha_{f_{D}}$. The parameters that describe the localization of these linear features can be estimated from the Radon transform of the time-frequency representation. ${ }^{12,13}$

The Radon transform of the time-frequency representation is defined as

$$
Q\left(\alpha_{f_{D}}, \nu_{D}\right)=\int_{x=-\infty}^{+\infty} P\left(x, f_{D}=\nu_{D}+\alpha_{f_{D}} x\right) d x
$$

The ridges have been mapped to peaks that are localized at the intercept and direction corresponding to the parameters of the point scatterers. The location of the peaks in the Radon-Wigner transform can now be used to estimate the cross-range position of the point scatterers. Here, we demonstrate an iterative algorithm for robust estimation of the scatterer positions from the parameters of the chirp components in the signal. The algorithm can be considered a modification of the CLEAN algorithm and the approach taken here is similar 
(a)

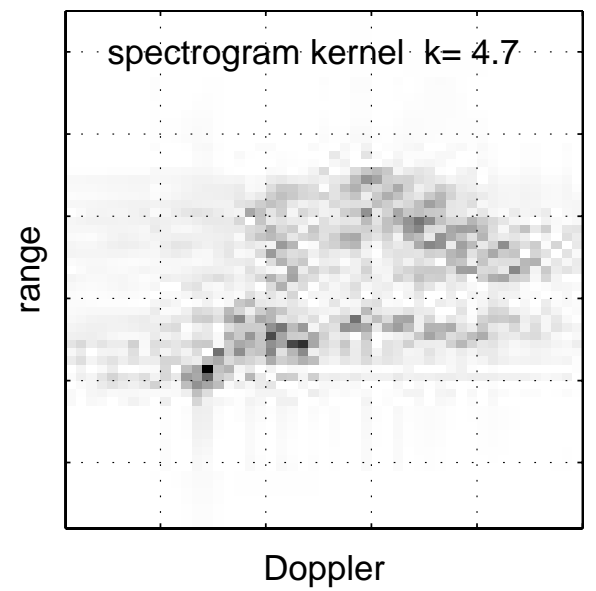

(c)

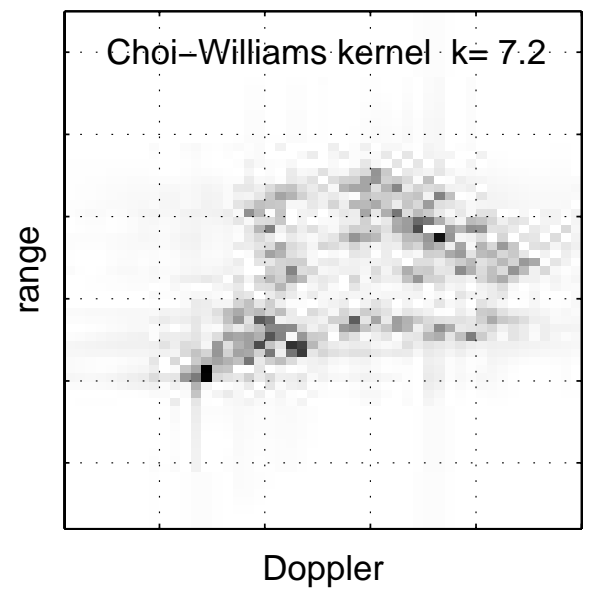

(b)

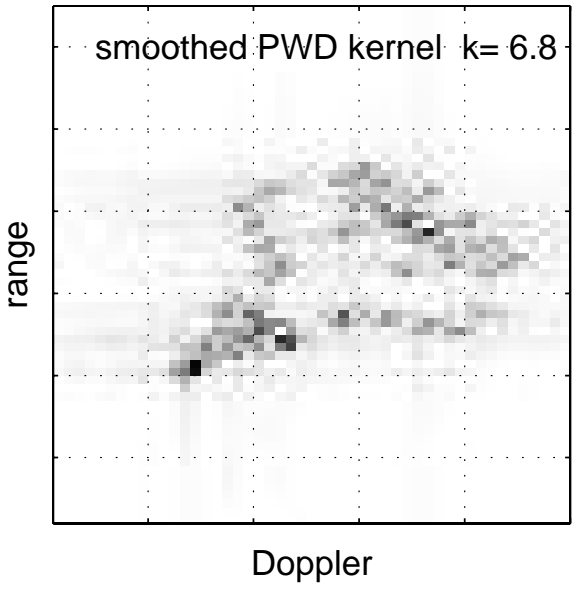

(d)

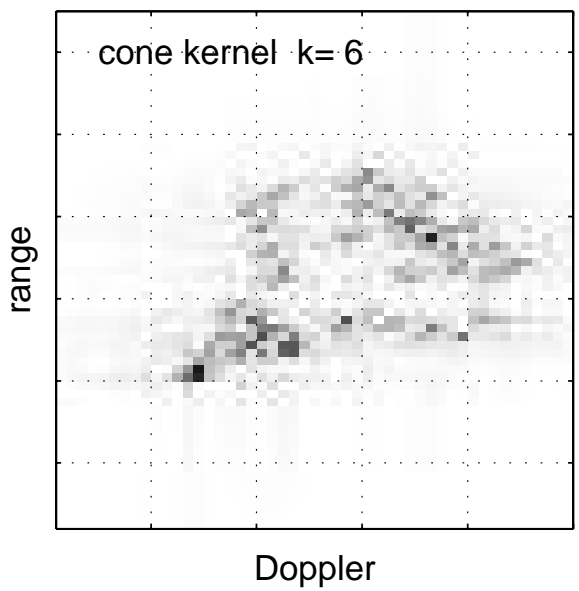

Figure 5. ISAR images after using flight path estimations shown in Fig. 4 , (a) Spectrogram kernel LRT, (b) pseudo smoothed Wigner distribution kernel LRT, (c) Choi-Williams kernel LRT, (d) cone kernel LRT. 
to that of the AUTOCLEAN algorithm for autofocus ISAR motion compensation. ${ }^{14}$ The algorithm can be summarized as follows

- Step 1: Compute the Radon-Wigner representation of the radar return signal $y\left(x, r=r_{i}\right)$.

- Step 2: Estimate the Doppler frequency change rate $\alpha_{f_{D}}$ of the highest peak in the Radon-Wigner representation.

- Step 3: De-chirp the signal by multiplication of $y(x)$ with the complex conjugate of a signal $y_{c}$ with chirp rate $\alpha_{f_{D}}$, i.e., $y_{c}(x)=e^{j 2 \pi \alpha_{f_{D}}} x^{2}$.

- Step 4: Estimate $f_{D}(x=0)$ through estimation of the frequency of the dominant sinusoid in the de-chirped signal (e.g. via the 1-D FFT of the de-chirped signal with zero-padding for high accuracy).

- Step 5: Subtract the dominant sinusoid from the de-chirped signal.

- Step 6: Undo the de-chirping by multiplication with $y_{c}^{*}(x)$.

- Step 7: Repeat Steps 1 to 6 until the relative change of the energy of the signal between two consecutive iterations is less than some threshold $\epsilon$.

A numerical example to demonstrate the algorithm is shown in Fig. 6. Figures 6a-d show the model and estimated point scatterer location, the input radar return signal and the remainder after subtraction of the first chirp component, the time-frequency representation of the radar return signal, and the Radon-Wigner transform of the input signal, respectively. Figures $6 \mathrm{e}-\mathrm{h}$ show the results at the second iteration of the algorithm. The result of the algorithm for a more complicated target is shown in Fig. 6. Figure 6a shows the an ISAR image that has been blurred by non-uniform - constant acceleration - target rotation. The Radon-Wigner chirp CLEAN algorithm in Fig. 6 results in a much improved localization of the scatterers.

\section{CONCLUSIONS}

In this paper we have demonstrated the use of the Radon transformation for ISAR range and Doppler frequency alignment. Accuracy of range alignment could be improved by using quadratic local Radon transformations, compared to the results that can be obtained using the sliding-window Radon transformation. Similar results have been obtained in experiments with measured data. For Doppler frequency alignment we have outlined an algorithm that is based on chirp detection in the Radon-Wigner transform. The algorithm is based on the linear Radon transform of the time-frequency representation. Consequently, the best results are obtained when scatterers are expressed as linear chirps in the time-frequency representation, e.g. for the case of constant acceleration of rotation that was demonstrated here. However, for more complicated target rotation similar results may be obtained using a generalized Radon-Wigner transformation for the detection of dominant scatterers in the time-frequency representation.

\section{REFERENCES}

1. P. Steeghs, S. Gelsema, and L. Kester, "Radon transforms for ISAR motion compensation and imaging," in Proc. of SPIE Conference on wavelets and independent component analysis applications IX, Vol. 4738, pp. 252-263, SPIE, 2002.

2. J. Li and P. Stoica, "Efficient mixed-spectrum estimation with applications to target feature extraction," IEEE Transactions on Signal Processing 44(2), pp. 281-295, 1996.

3. V. Chen and W. Miceli, "Simulation of ISAR imaging of moving targets," IEE Proc. Radar, Sonar and Navig. 148(3), pp. 160-166, 2001.

4. V. Chen and W. Miceli, "The effect of roll, pitch and yaw motions on ISAR imaging," in Proc. of SPIE Conference on Radar Processing, Technology, and Applications, IV, Denver, Colorado, Vol. 3810, pp. 149158, SPIE, 1999.

5. D. Wehner, High Resolution Radar Imaging, Artech House, Dedham MA, 1981. 
(a)

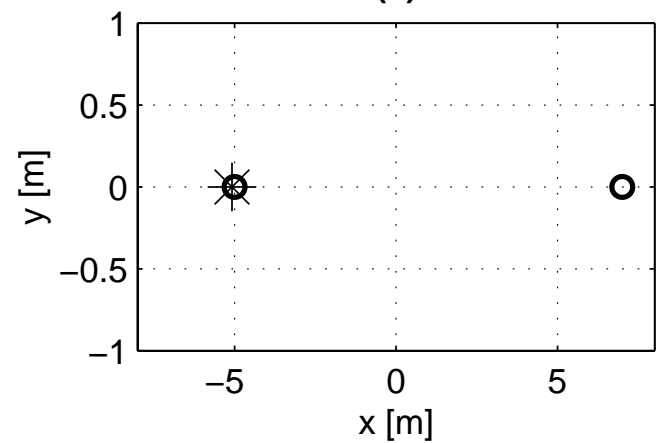

(c)

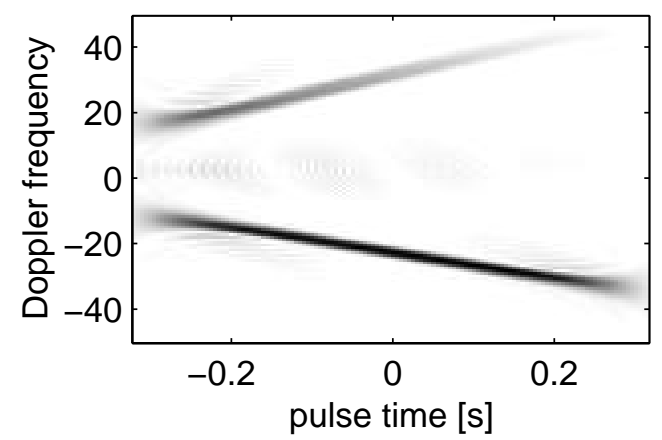

(e)

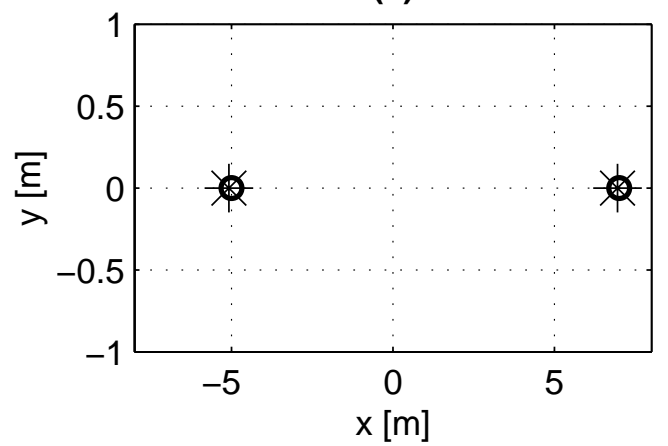

(g)

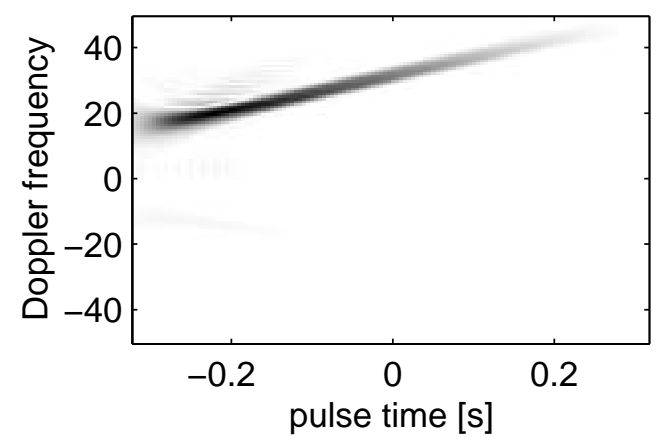

(b)

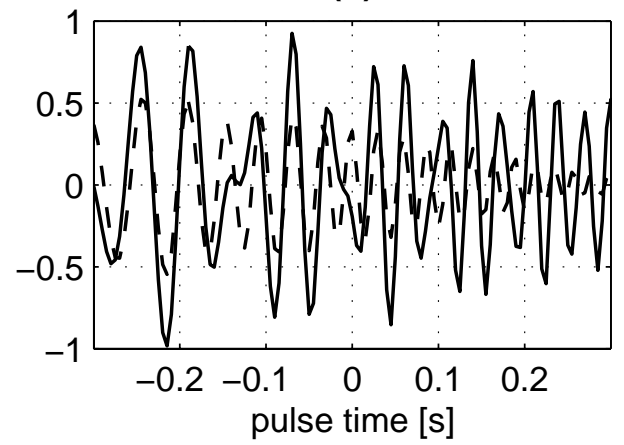

(d)

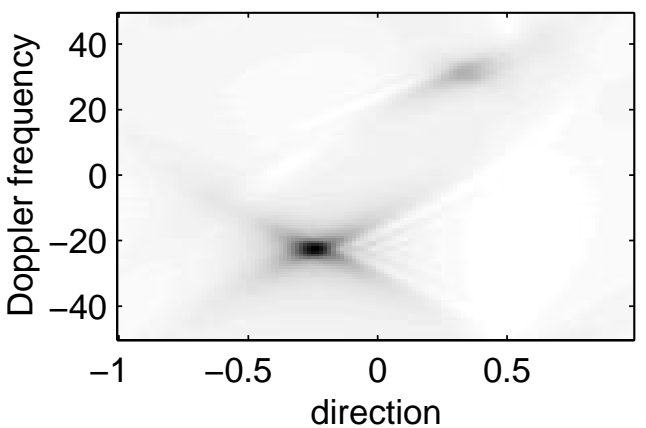

(f)

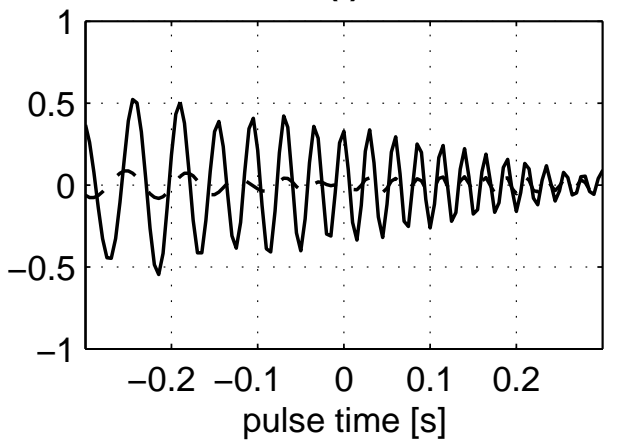

(h)

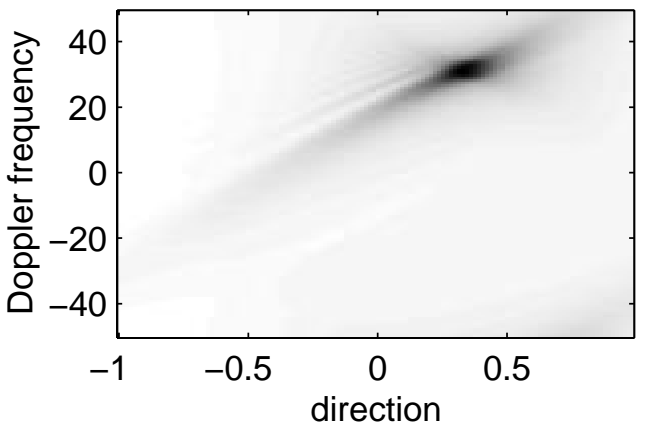

Figure 6. (a) model (circle) and result (star) of after one iteration, (b) input signal and (dashed line) remainder, (c) time-frequency representation of signal in (b), (d) Radon-Wigner representation of (c), (e) model and result after two iterations, input signal and remainder (dashed line) after second iteration, (f) time-frequency representation of signal in (d), and (g) Radon-Wigner representation of (f). 
(a)

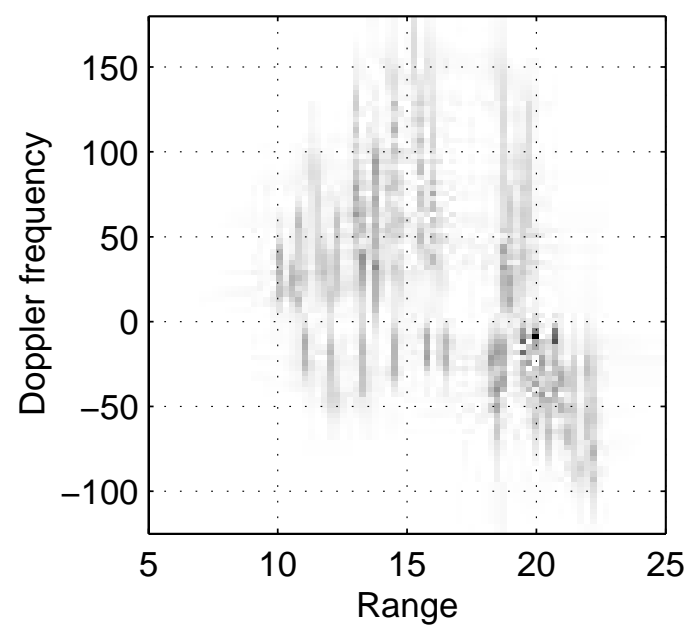

(b)

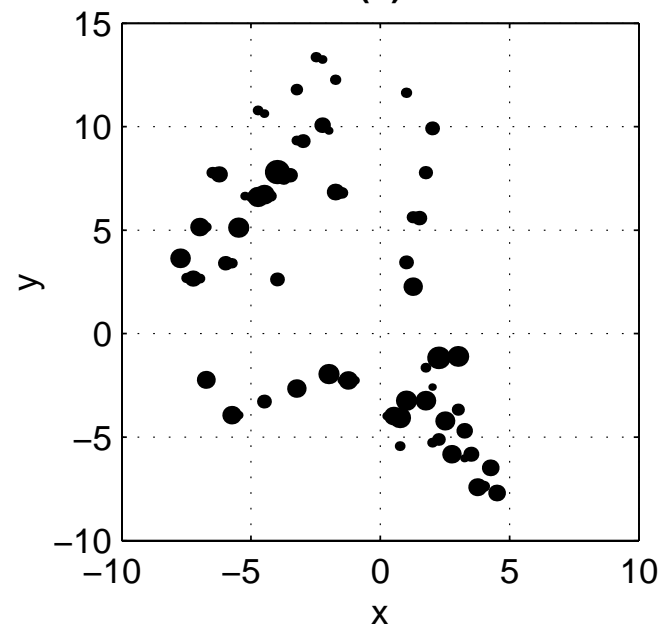

Figure 7. (a) Blurred ISAR image, due to non-linear (quadratic) rotation angle and (b) result of RW chirp CLEAN algorithm.

6. T. Sauer and A. Schroth, "Robust range alignment algorithm via Hough transform in an ISAR imaging system," IEEE Trans. on Aerospace and Electronic Systems 31(3), pp. 1173-1177, 1995.

7. L. Cohen, Time-Frequency Analysis, Prentice Hall, 1995.

8. P. Steeghs, "The Wigner-Radon representation," in Proceedings of the IEEE-SP Symposium on TimeFrequency and Time-Scale Analysis, Pittsburgh, PA, pp. 433-466, 1998.

9. P. Steeghs, R. Baraniuk, and J. Odegard, "Time-Frequency analysis of seismic reflection data," in Applications in Time-Frequency signal processing, A. Papandreou-Suppappola, ed., pp. 307-338, CRC Press, 2002.

10. V. Chen and S. Qian, "Joint Time-Frequency transform for radar range-Doppler imaging," IEEE Trans. on Aerospace and Electronic Systems 34(2), pp. 486-499, 1998.

11. V. Chen and H. Ling, Time-Frequency Transforms for Radar Imaging and Signal Analysis, Artech House, 2002.

12. J. Wood and D. Barry, "Radon transformation of time-frequency distributions for analysis of multicomponent signals," IEEE Trans. on Signal Processing 42(11), pp. 3166-3177, 1994.

13. V. Chen, "Applications of rotational time-frequency transforms to radar imaging of moving targets," in Proc. of SPIE Conference on wavelets and independent component analysis applications IX, Vol. 4738, pp. 241-251, SPIE, 2002.

14. J. Li, R. Wu, and V. Chen, "A robust autofocus algorithm for ISAR imaging of moving targets," in Proc. of SPIE, Algorithms for Synthetic Aperture Radar Imagery VII, ed. E. Zelnio, Vol. 4053, pp. 199-210, SPIE, 2000. 